\title{
METODOLOGIAS PARA INSERÇÃO DA ENGENHARIA ENGAJADA NA FORMAÇÃO UNIVERSITÁRIA DE ENGENHEIROS EM CONJUNTO COM A PROMOÇÃO DE ATIVIDADES DE EXTENSÃO
}

DOI: 10.37702/2175-957X.COBENGE.2021.3530

SANDRA RUFINO - ssrufino@yahoo.com.br

Universidade Federal do Rio Grande do Norte Avenida Gustavo Guedes 1789

59078-380 - Natal - RN

ANA VICTÓRIA CARLOS ALMEIDA - ana_victoriacarlos@hotmail.com UNIVERSIDADE FEDERAL DO RIO GRANDE DO NORTE

RUA FRANCISCO DE ASSIS FERNANDES 202

59780-000 - CARAÚBAS - RN

Matheus Henrique Silva Santos - matthew.santos123@outlook.com Universidade Federal do Rio Grande do Norte Rua Aderbal de Figueiredo, Casa 04, Resindência Universitária Biomédica Casa 04 59010-115 - Natal - RN

Marina Moura Lima - mmoura583@gmail.com Universidade Federal do Rio Grande do Norte Rua Teatrólogo Meira Pires 1993 59080-090 - Natal - RN

Arthur Tauan da Fonseca - arthurtauan23@hotmail.com Universidade Federal do Rio Grande do Norte Avenida Manoel Pacapara 574 59650-000 - Açu - RN

Tábata Juliany Moreira Silva - tabatajuliany@hotmail.com Universidade Federal do Rio Grande do Norte Rua Professor Leonor de França 03 59090-314 - Natal - RN

Resumo: $O$ ensino de engenharia é, majoritariamente, essencialmente teórico e 
tecnicista, visando a formação de engenheiros e engenheiras que idealizem e executem planos nacionais, os quais, em sua maioria, não incluem pessoas em vulnerabilidade social. Desta forma, para que os interesses sociais sejam includentes urge que no plano pedagógico dos cursos de engenharia sejam inseridas disciplinas de cunho humanista e que favoreçam práticas integradas à sociedade. Nesse sentido, o presente ensaio tem seguimento a partir da seguinte questão norteadora: "Como inserir a engenharia engajada na formação universitária de engenheiros em conjunto com a promoção de atividades de extensão?". O ensaio foi elaborado a partir de um levantamento qualitativo de alternativas para inserção da engenharia engajada e da extensão universitária no projeto pedagógico dos cursos de engenharia. O processo de elaboração deste trabalho foi dividido em três etapas: síntese dos principais conteúdos embasadores do tema, análise do referencial teórico e estruturação da discussão. E seu corpo está dividido em cinco sessões (introdução, metodologia, apresentação da problemática, soluções e considerações finais). Entende-se que a Engenharia Engajada (EE) é uma reivindicação de mudança social através de um novo papel das engenharias e sua inserção na formação universitária por se dar por meio de algumas vertentes: Enfoque CTS; aprendizagem focada em problemas; design criativo e extensão universitária. Constatou-se, portanto, que apesar do incentivo normativo e institucional para inserção de pautas sociais no plano de disciplinas das engenharias, ainda são ínfimas as instituições que as adotam, tornando a extensão uma boa alternativa para tal problemática.

Palavras-chave: Engenharia Engajada. Extensão Universitária. Formação em Engenharia 


\section{(c) COBENCE \\ METODOLOGIAS PARA INSERÇÃO DA ENGENHARIA ENGAJADA NA FORMAÇÃO UNIVERSITÁRIA DE ENGENHEIROS EM CONJUNTO COM A PROMOÇÃO DE ATIVIDADES DE EXTENSÃO}

\section{INTRODUÇÃO}

O ensino de engenharia é, em grande parte das instituições, essencialmente teórico e tecnicista, visando a formação de engenheiros e engenheiras que idealizem e executem planos nacionais, os quais, em sua maioria, não incluem pessoas em vulnerabilidade social. O Conselho Federal de Engenharia e Agronomia (CONFEA) caracteriza no art. $1^{\circ}$ da Lei $n^{\circ}$ 5.194/66 as atribuições do engenheiro pelas realizações de interesse social e humano que importem na execução de empreendimentos. Desta forma, para que os interesses sociais sejam includentes urge que no plano pedagógico dos cursos de engenharia sejam inseridas disciplinas de cunho humanista e que favoreçam práticas integradas à sociedade, explorando a empatia e o aprendizado dentro de uma engenharia engajada.

A formação universitária atualmente se baseia nos eixos da pesquisa, ensino e extensão. Enquanto os dois primeiros focam em aspectos mais teóricos formando profissionais tecnicistas, o último permite a prática e o contato dos discentes com a sociedade, favorecendo, desta forma, a valorização e inclusão do ser humano. Nesse sentido, o presente ensaio tem seguimento a partir da seguinte questão norteadora: "Como inserir a engenharia engajada na formação universitária de engenheiros em conjunto com a promoção de atividades de extensão?".

Com base nessa indagação, pode-se justificar o tema como sendo pertinente a se refletir, uma vez que estão sendo reformulados atualmente os projetos pedagógicos para os cursos de engenharia. Além disso, as possíveis respostas à questão norteadora implicam em mudanças através de uma inserção de aspectos sociais na formação e atuação de engenheiros e engenheiras.

\section{METODOLOGIA}

O objetivo deste ensaio é fazer um levantamento qualitativo de alternativas para inserção da engenharia engajada e da extensão universitária no projeto pedagógico dos cursos de engenharia, o qual sofre com os impactos causados pela atual formação focada no tecnicismo. O processo de elaboração deste trabalho foi dividido em três etapas: síntese dos principais conteúdos embasadores do tema, análise do referencial teórico e estruturação da discussão.

No primeiro momento, foi realizada uma pesquisa de literatura, de cunho exploratório, buscando materiais que abordassem a atual estrutura curricular da engenharia, as alternativas para desenvolvimento de uma formação voltada para intervenção e desenvolvimento social e o papel da extensão na formação acadêmica. Em seguida, na segunda etapa, realizou-se uma interpretação das obras que se mostraram relevantes para a estruturação do artigo. Por fim, na última etapa, produziu-se o presente ensaio.

Visando uma melhor compreensão das discussões propostas, o presente trabalho está dividido em cinco seções, sendo a primeira delas a introdução, seguida pela metodologia. Posteriormente, tem-se a explanação da problemática norteadora do ensaio, o atual modelo de ensino da engenharia, seguido, na quarta seção, pela apresentação das principais alternativas (soluções) para uma formação mais crítica e social. Por fim, têm-se as considerações finais acerca das questões levantadas no decorrer da produção. 


\section{APRESENTAÇÃO DA PROBLEMÁTICA}

O capitalismo, em sua função primordial - gerar lucros - tem deturpado as premissas da educação, alimentando uma competição acirrada para a formação acadêmica e técnica dos mais afortunados a ocuparem as posições econômicas mais elevadas no mercado de trabalho. Em virtude disso, a educação tem perdido seu caráter libertador, tornando-se apenas mais uma ferramenta do suposto sistema opressor a qual deveria se rebelar. Não fugindo a essa realidade intransigente, as engenharias, em um todo, têm guiado seus ensinos e grades curriculares segundo uma ótica econômica.

No final do século XX, Kawamura (1986) listou, como a engenharia deveria agir e seus três vieses da engenharia existentes, que competiam por espaço. O primeiro deles, seria uma engenharia elitista, voltado para mercado, completamente tecnocrata, enquanto o segundo baseia-se em ideias nacionais desenvolvimentistas e, o terceiro, uma engenharia preocupada com as causas sociais e políticas do contexto no qual está inserido.

Obviamente, com a dominação do capitalismo, a engenharia, tomada pelo alto teor técnico e científico de seus componentes curriculares e vazio de reflexões e debates políticos e sociais, como aponta Johnson e Wetmore (2008), tornou-se quase que puramente elitista, atrelada somente a questões econômicas. Ressalta-se, no entanto, que essa problemática não é uma mazela da contemporaneidade. Marx (2008), em "O Capital", livro escrito no século XIX, narrou que a engenharia e seus profissionais estavam comprometidos com os mais ricos, os donos das fábricas, desenvolvendo máquinas capazes de aumentar a produção de mais valia, mas que, concomitantemente, desqualificaram os trabalhadores, gerando desempregos.

A terceira vertente da engenharia apontada por Kawamura (1986), atrelada à militância, que era marginalizada no Brasil e no mundo nas décadas de 1970 e 1980, cresceu muito devido à crítica à neutralidade da Ciência e da Tecnologia, feitos pelos Estudos Sociais da Ciência e da Tecnologia (HERNÁNDEZ; KREIMER, 2011). Com isso, esse viés conquistou um pouco de espaço dentro das universidades (THIOLLENT; ALVEAR, 2009). A partir daí, cresce o número de movimentos que buscam transmutar a realidade social por meio de uma ótica da engenharia, a qual passou a ser chamada de Engenharia Engajada (EE).

Reforçando a necessidade do papel social da engenharia, a Resolução $n^{\circ} 2$, de 24 de abril de 2019 que institui as Diretrizes Curriculares Nacionais do Curso de Graduação em Engenharia, definidas pelo Conselho Nacional de Educação Superior, vinculado ao Ministério da Educação, define o perfil do egresso de engenharia como:

Art. $3^{\circ} \mathrm{O}$ perfil do egresso do curso de graduação em Engenharia deve compreender, entre outras, as seguintes características: I - ter visão holística e humanista, ser crítico, reflexivo, criativo, cooperativo e ético e com forte formação técnica; II - estar apto a pesquisar, desenvolver, adaptar e utilizar novas tecnologias, com atuação inovadora e empreendedora; III - ser capaz de reconhecer as necessidades dos usuários, formular, analisar e resolver, de forma criativa, os problemas de Engenharia; IV - adotar perspectivas multidisciplinares e transdisciplinares em sua prática; $\mathrm{V}$ - considerar os aspectos globais, políticos, econômicos, sociais, ambientais, culturais e de segurança e saúde no trabalho; VI - atuar com isenção e comprometimento com a responsabilidade social e com o desenvolvimento sustentável (CNE, 2019, p. 43)

No que diz respeito aos termos de diretrizes educacionais, o relatório da UNESCO Engineering: Issues, Challenges and Opportunities for Development (UNESCO, 2010) pede a urgência de uma renovação da formação em engenharia, no âmbito interno, através de 
reformas institucionais e curriculares, e no âmbito externo, a partir de uma contribuição mais efetiva na solução de problemas contemporâneos.

O relatório do World Congress on Engineering Education (WFEO), que fala sobre as recomendações acerca da EE nas engenharias, traz a responsabilidade social na engenharia com a inclusão de elementos normativos como justiça social, erradicação da pobreza e sustentabilidade ambiental. É importante salientar que projetos de cunho social demonstram ter impacto significativo no desenvolvimento de habilidades de design de protótipos por estudantes, conforme o estudo Prototype to Production: Processes and Conditions for Preparing the Engineer of 2020 (MCKENNA et al., 2011).

Mas quais as condições para que as mudanças demandadas pela EE sejam implementadas em escolas de engenharia? Entre os condicionantes para o sucesso de programas de inovação da educação em engenharia, o estudo Excelence in Engineering Education destaca: liderança, engajamento, design educacional, forma de planejamento e estratégia de implementação (GRAHAM, 2012).

Um dos desafios é a própria cultura epistêmica de profissionais da educação em engenharia que, geralmente, é socializada por uma matriz conservadora do modelo de ensino e aprendizagem e resistente a mudanças. As experiências empíricas demonstram uma grande variabilidade na inserção institucional da EE. Em alguns casos não se criam cursos, mas há uma integração de disciplinas de cursos já existentes.

No âmbito de concepções pedagógicas, teóricas e metodológicas, existem diversas vertentes. Estas, independentes da EE, funcionam como ferramentas de aprendizagem a serem combinadas entre si ou não, em cada experiência singular. Podemos destacar aqui a contribuição dos estudos CTS, que refletem criticamente sobre temas como o determinismo tecnológico, a neutralidade da técnica e a preponderância dos interesses industriais na produção sociotécnica (BAZZO; PEREIRA; LINSINGEN, 2008).

É necessário, não somente a atuação de profissionais da própria instituição na liderança de um processo de transformação do modelo educacional, mas também o apoio das instâncias decisórias, assim como um suporte administrativo e financeiro. Este conjunto de elementos deve obter volume suficiente que possibilite espaços para a introdução de projetos de mudança curricular, acesso a laboratórios e sua equipagem e fomento a projetos de extensão.

Portanto, as iniciativas de EE podem usar um ou mais enfoques como ferramentas para a aprendizagem de reflexão e as habilidades necessárias de engenheiras e engenheiros. O que diz respeito à EE é que ela demanda um conjunto particular de ações transformativas do mundo real. Dessa forma, nas instituições acadêmicas a EE sempre será vinculada à extensão.

\section{SOLUÇÕES}

Para que a formação do engenheiro aconteça de uma maneira mais humanística e com a intenção de que se tenha mais profissionais com uma visão crítica quando se trata das relações sociais e ambientais (FERREIRA et al., 2017), a incorporação de elementos que estejam envolvidos com a Ciência, Tecnologia e Sociedade (CTS) se fazem necessários; com o objetivo de integrar a educação científica, tecnológica e social.

Esta inclusão pode ser feita através de modificações nas grades curriculares dos cursos, seja por meio de disciplinas ou através da inserção de projetos de extensão, agregando ao aprendizado desenvolvido, novas competências, ações e valores, implicando em mudanças na transferência de saberes.

Segundo Dwek; Coutinho e Matheus (2011) a introdução do enfoque de CTS nas grades curriculares dos cursos de engenharia agregaria significativamente a formação do 
engenheiro, que teria uma visão mais crítica e diálogos mais abrangentes nas discussões sobre os caminhos da tecnologia. De acordo com os mesmos autores, a visão separatista entre o que seria os estudos da engenharia e as ciências humanas não pode permanecer, pois se configura como desvantajoso a ambas as áreas de conhecimento.

Nesse cenário, convém falar sobre o que seria a Engenharia Engajada, conceito esse que se atrela bastante à ideia da presença de CTS nos cursos de engenharia. A Engenharia Engajada surge como uma opção para que essa formação engessada e pautada somente em aspectos técnicos e racionais diminua, além de permitir que o engenheiro possa vir a se tornar um profissional com um olhar mais sensível para as diversas questões que permeiam tanto o seu ambiente de trabalho quanto o seu entorno. A formação universitária é, portanto, redesenhada, passando a ter além do técnico, um viés social revestido com valores humanísticos e éticos.

Segundo Kleba (2017), pode-se dizer que EE é uma reivindicação de mudança social através de um novo papel das engenharias. De acordo com o mesmo autor, a EE se apresenta em ações de desenvolvimento inclusivo, diretrizes de educação, organizações sem fins lucrativos, negócios sociais e redes de tecnologias sociais, entre outros. Ele ainda cita alguns exemplos dessa produção sociotécnica na engenharia, tais como a ênfase em projetos que impactem a vida de grupos sociais marginalizados; a integração de ações sociais e da extensão no ensino e na pesquisa; bem como novas vertentes pedagógicas que possibilitem essa mudança social.

A Engenharia Engajada pode-se dar de diferentes formas dentro da formação dos futuros profissionais de engenharia. Kleba (2017) afirma que ela pode ser implementada tanto no design institucional, quanto nas concepções pedagógicas. Cita-se alguns espaços para a criação de EE nas escolas de engenharia, tais como: projetos-piloto de mudança curricular, integração de disciplinas de cursos já existentes ou até mesmo a criação de novos cursos, além de, claro, projetos de extensão.

Algumas vertentes debatidas por Kleba (2017) podem auxiliar na inserção da engenharia engajada na formação universitária, especial no âmbito das concepções pedagógicas, teóricas e metodológicas, são elas:

- Uso do enfoque CTS: metodologia que permite ao professor inserir alternativas de ensino que possam contribuir para o processo de formação crítica dos indivíduos. Aikenhead e Solomon (1994) discorre que as estratégias do ensino com esse enfoque envolvem conteúdos com relevância social, permitindo assim a problematização destes e o estabelecimento de relações socioambientais;

- Aprendizagem focada em problemas - problem based learning (PBL): metodologia de ensino que se opõe ao modelo vigente na qual são expostos conteúdos fragmentados e rapidamente esquecidos. Nela é selecionado um problema complexo, para o qual são estudadas soluções, integrando as mais diversas disciplinas e tendo como resultado a solução da problemática;

- Design criativo: metodologia que objetiva estimular a inovação através de desenhos e soluções através de atividades práticas. Este pode ser aliado à um PBL e obtendo a resolução do problema através de protótipos ou desenhos.

Por fim, uma vertente de grande aplicação da engenharia engajada na universidade, e em particular nas engenharias, é a extensão universitária, que segundo o artigo $2^{\circ}$ da Resolução Nº 077/2017-CONSEPE UFRN (2017, sem página), é:

Processo educativo, cultural e científico que articula o ensino e a pesquisa de forma indissociável para viabilizar relações transformadoras entre a 
Universidade e a sociedade, a partir de um diálogo que envolva os diferentes saberes (das ciências, das tecnologias, das artes, das humanidades e da tradição), permitindo novas criações, socializações e mudanças recíprocas, com o envolvimento e inserção de alunos, professores e técnicos administrativos em experiências reais junto a diferentes grupos e populações que com elas interagem, por meio de ações: I) projetos; II) programas.

Considerando que a extensão é um processo educativo e científico, ao praticá-la está se produzindo um conhecimento que potencializa a relação de transformação entre a Universidade e a Sociedade (SERRANO, 2010). Ela se insere como um meio transformador tanto para a comunidade onde irá ocorrer a ação como para quem participa dela atrelando conhecimentos acadêmicos com os saberes populares.

Sabendo da importância da extensão na formação, algumas universidades, a exemplo da Universidade Federal do Rio Grande do Norte (UFRN), tornaram-na parte integrante e obrigatória dos projetos pedagógicos de todos os cursos de graduação. No caso em específico da UFRN, através do Art 6 ${ }^{\circ}$, Resolução № 038/2019-CONSEPE, de 23 de abril de 2019, foi determinada a sua obrigatoriedade em um percentual mínimo de $10 \%$ (dez por cento) da carga horária total do curso de atividades extensionistas. Sendo essa uma conquista de imensa importância para a vida acadêmica e formação daqueles que participam dessa experiência única.

Portanto, a extensão pode ser utilizada como aliada na inserção da Engenharia Engajada na universidade através da promoção de projetos que possibilitem a socialização entre docentes, discentes e o público externo. A medida que propõe a resolução de problemas sociais e ambientais, a discussão acerca de temáticas importantes, o debate de como difundi-los ao meio estudantil, o estímulo à cultura e cidadania.

Essas ações de extensão podem se dar por meio de palestras; oficinas abertas ao público externo; cursos profissionalizantes ministrados pelo próprio corpo docente e discente; apresentações culturais; aulas que promovam a busca por uma melhor qualidade de vida e saúde. Dentre inúmeras outras atividades que possam integrar o público interno e externo da universidade, possibilitando assim o conhecimento das mais diversas camadas sociais presentes na sociedade.

\section{CONSIDERAÇÕES FINAIS}

Conforme observado no presente trabalho, constatou-se, por meio de revisão bibliográfica, que apesar do incentivo normativo e institucional para inserção de pautas sociais presentes nas Diretrizes Curriculares Nacionais do Curso de Graduação em Engenharia e nas normativas da UNESCO no que cerne a educação, ainda são ínfimas as instituições que adotam componentes curriculares mais humanísticos e incentivam atividades práticas junto à comunidade.

Assim, entende-se que com a parca oferta e inserção de disciplinas que instiguem o pensamento crítico, inclusivo e visando soluções acessíveis, o desenvolvimento de atividades de extensão surge como uma alternativa. Uma vez que o próprio contato com a sociedade proporciona a reflexão dos discentes a partir do contato com realidades diferentes das suas, ou seja, têm na prática as vivências que seriam comentadas apenas a nível teórico em sala de aula.

Portanto, tendo em vista os impactos positivos para academia e sociedade como um todo da formação em engenharia baseada na engenharia engajada e usufruindo do pilar da extensão, urge a previsão obrigatória de certas disciplinas da área na Diretriz Curricular de ensino, bem como uma maior discussão sobre a temática.th 
O tema dá ainda abertura para realização de mais pesquisas no âmbito do estudo de possíveis impactos consequentes da execução específica de práticas de extensão e engenharia engajada, como sugerido ao longo do texto. Podendo inclusive ser feita uma análise comparativa entre discentes expostos à atividades práticas exploratórias, como a extensão e àqueles expostos somente ao ensino e pesquisa.

\section{REFERÊNCIAS}

AIKENHEAD, Glen; SOLOMON, Joan. STS education: international perspectives on reform. Ways of knowing science series. New York: Teachers College Press, 1994, p. 4759.

BAZZO, Walter Antonio; PEREIRA, Luiz Teixeira do Vale; LINSINGEN, Irlan Von. Educação tecnológica: enfoques para o ensino de engenharia. 2.ed. Florianópolis: Ed. da UFSC, 2008.

CONSELHO NACIONAL DE EDUCAÇÃO CÂMARA DE EDUCAÇÃO SUPERIOR. CNE. RESOLUÇÃO $n^{\circ}$ 2, de 24 de abril de 2019. Dispõe sobre as diretrizes curriculares nacionais do curso de graduação em engenharia. Diário Oficial da União, Brasília, 24 de abril de 2019. Seção 1, p. 43.

DE GRAAFF, Erik; GUERRA, Aida; KOLMOS, Anette; AREXOLALEIBA, Nestor. Global research community: Collaboration and Developments. Aalborg: Aalborg University Press, 538p., 2015.

DWEK, Maurício; COUTINHO, Heloísa; MATHEUS, Fernando. Por uma formação crítica em engenharia. In: XXXIX Congresso Brasileiro de Educação em Engenharia, 2011, Blumenau. Anais. Blumenau. Disponível em: http://www.abenge.org.br/cobenge/legado/arquivos/8/sessoestec/art2032.pdf. Acesso em 15 abr. 2021.

FERREIRA, Marta Lucia Azevedo; SOUZA, Cristina Gomes de; SPRITZER, Ilda Maria de Paiva Almeida; CHRISPINO, Álvaro. Contribuições da abordagem CTS para a formação em engenharia no Brasil. Revista Espacios. v. 38, n. 20, p.33-46. 2017.

GRAHAM, Ruth. Achieving excellence in engineering education: the ingredients of successful change. The Royal Academy of Engineering and MIT, London, March 2012.

HERNÁNDEZ, Antonio Arellano; KREIMER, Pablo. Introducción General. Estudio social de la ciencia y la tecnología desde América Latina. Siglo del Hombre Editores, 2011.

JOHNSON, Deborah G.; WETMORE, Jameson M. STS and ethics: Implications for engineering ethics. The handbook of science and technology studies, $3 \mathrm{ed}$. Cambridge (MA). MIT Press, 2008.

KLEBA, John Bernhard. Engenharia engajada: desafios de ensino e extensão. Revista Tecnologia e Sociedade., Curitiba, v. 13, n. 27, p. 170-187, jan./abr. 2017.

KAWAMURA, Lili Katsuco. Tecnologia e política na sociedade: engenheiros, reivindicações e poder. São Paulo. Editora Brasiliense, 1986.

MARX, Karl. O capital: crítica da Economia Política: livro I. 26a edição. Rio de Janeiro: Civilização Brasileira, 2008.

MCKENNA, Ann F.; OKUDAN-KREMER, Gull E.; PLUMB, Carolyn; RO, Hyun Kyoung; YIN, Alexander. Approaches to engaging students in engineering design and problem solving. Proceedings of the American Society for Engineering Education. In: ASEE Annual 
2011 Vancouver Anais. https://peer.asee.org/approaches-to-engaging-students-in-engineering-design-andproblem-solving. Acesso em 16 abr. 2021.

SERRANO, Rossana Maria Souto Maior. Conceitos de extensão universitária: um diálogo com Paulo Freire. Disponível em: http://www.prac.ufpb.br/copac/extelar/atividades/discurssao/conceitos_de_extensao_unive rsitaria.pdf: Acesso em: 18/04/2021.

THIOLLENT, Michel; ALVEAR, Celso Alexandre Souza de. Sobre o papel do engenheiro de produção em projetos sociais. In: XVI SIMPÓSIO DE ENGENHARIA DE PRODUÇÃOSIMPEP, 2009, Bauru. Anais. Bauru. Disponível em: https://simpep.feb.unesp.br/anais_simpep.php?e=4. Acesso em: 15 abr. 202

UNESCO. Engineering: Issues, Challenges and Opportunities for Development. Disponível em: Disponível em: http://unesdoc.unesco.org/images/0018/001897/189753e.pdf. Acesso em 16 abr. 2021.

UNIVERSIDADE FEDERAL DO RIO GRANDE DO NORTE. CONSEPE. Resolução $\mathbf{n}^{\circ}$ 077/2017, de 27 de junho de 2017. Dispõe sobre as modalidades e ações de extensão universitária na Universidade Federal do Rio Grande do Norte - UFRN. CONSEPE, 2017.

\title{
METHODOLOGIES FOR THE INSERTION OF ENGINEERING ENGAGED IN UNIVERSITY TRAINING OF ENGINEERS IN JOINT WITH THE PROMOTION OF EXTENSION ACTIVITIES
}

\begin{abstract}
Engineering education is, for the most part, essentially theoretical and technician, aiming at the training of engineers who devise and execute national plans, which, for the most part, do not include people in social vulnerability. Thus, in order for social interests to be included, it is urgent that the pedagogical plan of engineering courses includes disciplines of a humanistic nature and on behalf of practices integrated to society. For that matter, the present essay follows on from the following guiding question: "How to insert engaged engineering in the university education of engineers with the promotion of extension activities?". The essay was based on a qualitative survey of alternatives for inserting engaged engineering and university extension in the pedagogical project of engineering courses. The process of preparing this work was divided into three stages: synthesis of the main content underlying the theme, analysis of the theoretical framework and structuring of the discussion. And its body is divided into five sessions (introduction, methodology, presentation of the problem, solutions, and final considerations). It is understood that Engaged Engineering (EE) is a claim for social change through a new role for engineering and its insertion in university education because it takes place through some aspects: CTS approach; problem-focused learning; creative design and university extension. It was found, therefore, that despite the normative and institutional incentive to insert social guidelines in the engineering disciplines plan, the institutions that adopt them are still insignificant, making extension a good alternative for such a problem.
\end{abstract}

Keywords: Engaged Engineering. University Extension. Engineering Education. 